\title{
DESENVOLVIMENTO E \\ VALIDAÇÃO DE UMA ESCALA DIRETA PARA MENSURAÇÃO DE ATITUDES RELATIVAS A DESTINOS TURÍSTICOS
}

DEVELOPMENT AND VALIDATION OF A DIRECT SCALE FOR MEASURING ATTITUTES TOWARDS TOURISM DESTINATIONS

\section{DESARROLLO Y VALIDACIÓN DE UNA ESCALA DIRECTA PARA LA MENSURACIÓN DE LAS ACTITUDES RELATIVAS A DESTINOS TURÍSTICOS}

\section{Glauber Eduardo de Oliveira Santos}

Instituto Federal de Educação, Ciência e Tecnologia (IFSP) Doutor em Economia do Turismo e do Meio Ambiente, Universitat de les Illes Balears (UIB) glaubereduardo@gmail.com Janaina de Moura Engracia Giraldi Faculdade de Economia, Administração e Contabilidade de Ribeirão Preto (FEARP) da USP Doutora em Administração, USP jgiraldi@usp.br Data de Submissão: 11/08/2015 Data de Aprovação: 01/12/2015

RESUMO: A mensuração das atitudes é um dos principais desafios para a utilização desse conceito na explicação e na previsão do comportamento dos consumidores e dos turistas. Tal mensuração tem sido usualmente feita por meio de escalas indiretas, através das quais as atitudes são inferidas a partir de uma série de indicadores. Contudo, escalas diretas nas quais os indivíduos julgam suas próprias atitudes por meio de declarações abertamente avaliativas podem ser mais adequadas a determinadas circunstâncias, especialmente quando o objeto em questão é pouco conhecido pelo sujeito. Atualmente, poucas escalas diretas de atitudes estão disponíveis na literatura, formando uma base teórica e empírica frágil para a adoção dessa alternativa. Na tentativa de preencher esta lacuna, o presente estudo visa desenvolver uma escala direta para a mensuração das atitudes dos indivíduos em relação a destinos turísticos de lazer. Isto é feito por meio de um trabalho baseado no 
procedimento para desenvolvimento de escalas sugerido por DeVellis (2003) e composto por oito etapas distintas. $O$ instrumento desenvolvido foi empiricamente testado junto a uma amostra de estudantes. Os resultados obtidos apontam que as escalas diretas são adequadas para a mensuração das atitudes relativas a destinos turísticos. É evidenciado também que escalas diretas de único item são mais adequadas do que as de múltiplos itens.

Palavras-chave: Atitude. Destino turístico. Comportamento do consumidor.

ABSTRACT: Measuring attitudes is a major challenge to the adoption of this concept for explaining and forecasting the behavior of consumers and tourists. This type of measurement has traditionally been conducted through indirect scales, with attitudes being inferred based on a series of indicators. However, direct scales in which individuals judge their own attitudes, through openly evaluative statements, can be more appropriate in specific conditions, especially when the subject only has a superficial knowledge of the object in question. The literature has very few scales for measuring direct attitude, resulting in a fragile theoretical and empirical basis for the adoption of this measurement. To fill this gap, this study develops a direct scale for measuring attitudes towards leisure tourism destinations. It follows the eight-step procedure for scale development suggested by DeVellis (2003). The instrument was empirically tested in a sample of students. The results show that direct scales are adequate for measuring attitudes towards tourism destinations, and that direct single-item scales are better than multiple-item scales.

Keywords: Attitude. Tourism destination. Consumer behavior.

RESUMEN: La mensuración de las actitudes es uno de los principales retos para la utilización de ese concepto en la explicación y en la previsión del comportamiento de los consumidores y de los turistas. Tal mensuración ha sido realizada usualmente por medio de escalas indirectas, a través de las cuales se infieren las actitudes a partir de una serie de indicadores. Con todo, las escalas directas en las cuales los individuos juzgan sus propias actitudes por medio de declaraciones abiertamente valorativas pueden ser más adecuadas para determinadas circunstancias, especialmente cuando el objeto en cuestión es poco conocido por el sujeto. Actualmente hay pocas escalas directas de actitudes disponibles en la literatura, formando una base teórica y empírica frágil para la adopción de esa alternativa. En el intento de llenar ese vacío, el presente estudio tiene el propósito de desarrollar una escala directa para la mensuración de las actitudes de los individuos en relación a destinos turísticos de ocio. Esto se ha realizado por medio de un trabajo basado en el procedimiento para el desarrollo de escalas sugerido por DeVellis (2003) y compuesto por ocho etapas distintas. El instrumento desarrollado fue testeado empíricamente en una muestra de estudiantes. Los resultados obtenidos señalan que las escalas directas son adecuadas para la mensuración de las actitudes relativas a destinos turísticos. Se hace evidente también que las escalas directas de único ítem son más adecuadas que las de múltiples ítems.

Palabras clave: Actitud. Destino turístico. Comportamiento del consumidor. 


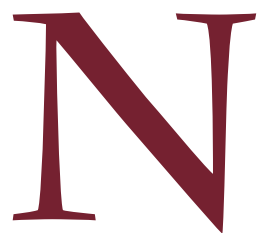

o sistema de mercado, o consumidor é livre para escolher suas viagens. Consequentemente, os destinos turísticos ocupam posição de dependência em relação às escolhas do consumidor. Por outro lado, o marketing oferece às organizações gestoras de destinos uma série de ideias e ferramentas capazes de influenciar tais escolhas. Desta forma, o marketing de destinos turísticos permite o desenvolvimento de estratégias para influenciar o consumidor a se comportar de forma vantajosa para o destino. Neste contexto, faz-se evidente a importância do estudo do comportamento do consumidor de turismo como forma de oferecer suporte teórico e empírico à análise de estratégias e suas prováveis consequências para o destino.

Um dos conceitos mais amplamente difundidos para a explicação do comportamento do consumidor em geral é a atitude, definida por Eagly e Chaiken (1993) como uma tendência psicológica de avaliação de uma entidade em alguma medida favorável ou desfavorável. Apesar de esta ser considerada uma definição clássica (ALBARRACÍN et al., 2005), trata-se de apenas uma das inúmeras existentes. Ajzen (2005) reconhece que existem muitas definições formais de atitude, de modo que o conceito apresenta contornos relativamente difusos. Contudo, o autor defende a existência de um consenso de que a característica essencial da atitude é sua natureza avaliativa. Neste sentido, a atitude em relação a um objeto específico pode ser representada por um único número que representa a posição do indivíduo em uma escala avaliativa que se estende entre os extremos negativo e positivo (FISHBEIN; AJZEN, 1975).

As atitudes têm sido utilizadas para explicar as ações dos indivíduos ao menos desde os anos 1920. A presença das atitudes na literatura sobre comportamento do consumidor é marcante, constituindo um dos temas mais importantes da psicologia social (FISHBEIN; AJZEN, 1975). O poder das atitudes na explicação e na previsão das escolhas de destinos turísticos também tem sido amplamente testado e confirmado por pesquisas empíricas (JALILVAND; EBRAHIMI; SAMIEI, 2013; JALILVAND et al., 2012; LEE, 2009; RAGHEB; TATE, 1993; UM; CROMPTON, 1990). 
Um dos principais desafios para a utilização do conceito de atitudes é sua mensuração. Uma vez que as atitudes pertencem ao mundo mental particular de cada indivíduo, elas não são diretamente observáveis. Portanto essa característica latente apenas pode ser estudada a partir de inferências feitas com base em sinais externos observáveis, tais como ações e declarações. Diferentes tipos de métodos têm sido utilizados para mensurar as atitudes, incluindo autorrelatos, técnicas de mensuração implícita e métodos não intrusivos. Dentre estes, as pesquisas baseadas em autorrelatos são certamente as mais frequentes (KROSNICK; JUDD; WITTENBRINK, 2005).

De acordo com Ajzen (2005), a mensuração das atitudes a partir de autorrelatos pode ser feita por meio de escalas diretas ou indiretas. As escalas diretas são aquelas em que os respondentes julgam suas próprias atitudes por meio de declarações abertamente avaliativas, como péssimo ou excelente e desejável ou indesejável. Escalas indiretas são aquelas baseadas em julgamentos relativos a diferentes aspectos do objeto em questão. Neste caso, a atitude em relação a um destino turístico poderia ser inferida a partir de julgamentos referentes a elementos objetivos, tais como atrativos naturais e culturais, serviços, preços, distância e outros. Poderiam, ainda, ser incluídos nessa lista emoções e intenções do indivíduo em relação ao destino.

A maior parte das escalas de atitudes desenvolvidas e apresentadas na literatura científica adota a perspectiva indireta principalmente por conta da preocupação com a confiabilidade da mensuração (DEVELLIS, 2003). Por outro lado, escalas diretas podem se mostrar vantajosas nos casos de atitudes relativas a objetos pouco conhecidos. Mas, apesar de sua utilidade, poucas escalas diretas de atitudes estão disponíveis na literatura, formando uma base teórica e empírica frágil para a adoção dessa alternativa. Além de menos frequentes, o grau de desenvolvimento das escalas diretas é bastante reduzido. Questões como confiabilidade e validade das poucas escalas disponíveis foram pouco estudadas, de forma que não se dispõe de referências sólidas para embasar estudos que precisem desses instrumentos. Na tentativa de preencher esta lacuna no contexto das atitudes relativas a destinos turísticos, o presente estudo visa desenvolver uma escala direta para a mensuração das atitudes dos indivíduos 
em relação a destinos turísticos de lazer. Isto é feito por meio de um trabalho baseado no procedimento para desenvolvimento de escalas sugerido por DeVellis (2003). Esse procedimento tem sido considerado padrão para a criação e teste de novas escalas (FARH; CANNELLA; LEE, 2006; RUNGTUSANATHAM; ANDERSON; DOOLEY, 1999), sendo composto por oito etapas organizadas em uma sequência lógica. Cada etapa tem diferentes objetivos e utiliza métodos distintos. Esse procedimento será detalhadamente apresentado e discutido adiante. $O$ instrumento desenvolvido foi empiricamente testado junto a uma amostra de estudantes de ensino superior de diversas áreas.

A seção a seguir traz um debate teórico sobre escalas diretas e indiretas de atitudes. Em seguida são discutidas as características formais de uma boa escala de atitudes. A quarta seção apresenta alguns dos principais estudos que utilizam escalas de atitudes relativas a destinos turísticos. A quinta seção apresenta o método e os resultados do estudo empírico realizado para o desenvolvimento de uma escala de atitudes relativas a destinos turísticos de lazer, sendo seguida pela conclusão.

\section{ESCALAS DIRETAS E INDIRETAS DE ATITUDES}

As escalas de atitude baseadas em autorrelatos podem ser classificadas em diretas ou indiretas (AJZEN, 2005). Pesquisas que utilizam escalas diretas pedem aos respondentes que avaliem abertamente o objeto em questão. Um exemplo de escala direta é aquela desenvolvida por Hsu, Cai e Li (2010), na qual o respondente deveria indicar, por exemplo, o quão agradável seria uma viagem para Hong Kong. Pesquisas que utilizam escalas indiretas solicitam aos respondentes avaliações relativas a diferentes aspectos do objeto em questão. O conjunto de aspectos a ser avaliado frequentemente inclui percepções acerca das características do objeto e emoções por ele despertadas. Um exemplo de escala indireta é aquela desenvolvida por Lee (2009) e que solicitava que o respondente indicasse, por exemplo, suas percepções relativas à conservação do meio ambiente do destino turístico e ao seu afeto por determinadas pessoas envolvidas na visitação deste.

A maior parte das escalas de atitudes desenvolvidas e apresentadas na literatura científica adota a perspectiva indireta. A origem dessa preferência parece residir 
na preocupação com a confiabilidade das escalas. Como as atitudes não podem ser diretamente observadas, sendo apenas objeto de inferências feitas a partir de indicadores, o risco de erro na mensuração está sempre presente. Uma forma de reduzir esse problema é a mensuração por meio múltiplos indicadores, de modo que os erros de medida de cada indicador compensem uns aos outros (DEVELLIS, 2003). Assim, a inferência feita a partir de várias medidas paralelas tende a se aproximar mais do verdadeiro valor da atitude. Esse tema é detalhadamente discutido na próxima seção deste artigo. O importante por ora é destacar que, a fim de ampliar a confiabilidade da escala de atitude relativa a um objeto, os pesquisadores comumente recorrem à abordagem indireta.

A diferença entre escalas diretas e indiretas também se faz presente na questão da dimensionalidade das relações do indivíduo com o objeto (FISHBEIN; AJZEN, 1975). Alguns objetos mantêm relações relativamente simples e diretas com os indivíduos, enquanto outros mantêm relações multidimensionais e complexas. A proteção do meio ambiente pode ser tida como exemplo de elemento com que desperta reações diversas nos indivíduos, envolvendo, dentre outros, a reciclagem de lixo domiciliar, a visitação de ambientes protegidos, a educação dos filhos e a seleção de candidatos para as eleições. Em casos como este as escalas diretas não são amplamente funcionais, já que diversos aspectos devem ser resgatados da memória para a avaliação do objeto. Perguntar ao indivíduo se a proteção do meio ambiente é algo bom ou ruim pode oferecer informações superficiais e menos consistentes do que aquelas oferecidas por escalas indiretas. Por outro lado, nesse quesito, objetos que despertam uma gama limitada de reações nos indivíduos são mais adequados ao uso de escalas diretas. Fishbein e Ajzen (1975) afirmam que diferentes formas de mensurar uma mesma dimensão tendem a produzir resultados bastante similares.

A composição de escalas indiretas para mensurar atitudes relativas a objetos que despertam reações multidimensionais é bastante complexa em razão da seleção dos elementos que devem fazer parte da escala. Esta questão se faz particularmente presente nas escalas indiretas de atitudes relativas a destinos turísticos. Uma breve comparação de escalas desse tipo disponíveis na literatura acadêmica (JALILVAND; EBRAHIMI; SAMIEI, 2013; JALILVAND et al., 2012; LEE, 2009; MOHSIN, 2005; SPARKS; PAN, 2009; UM; CROMPTON, 1990) revela 
enormes disparidades não apenas com respeito a elementos específicos de cada localidade, mas também com relação a aspectos mais gerais e supostamente comuns a todos os destinos. Portanto a construção de escalas de atitudes pela via indireta passa obrigatoriamente pelo debate de quais são os aspectos a serem incluídos. A inexistência de um conjunto indiscutivelmente adequado de aspectos adiciona uma dose de arbitrariedade inevitável aos resultados alcançados. Portanto, à medida que a dimensionalidade das reações despertadas pelo objeto cresce, as escalas indiretas correm maiores riscos se desviarem do foco de análise e acabarem avançando sobre outros construtos. A consequência disto seria a composição de escalas com baixo grau de validade (DEVELLIS, 2003).

Escalas diretas de atitudes podem ser mais vantajosas quando o grau de conhecimento dos indivíduos sobre o objeto é pequeno. Nestes casos, o indivíduo pode não ter opiniões sólidas acerca de cada elemento componente de uma escala indireta, fazendo com que as respostas oferecidas sejam pouco confiáveis. Em situações extremas, os indivíduos podem simplesmente não ter qualquer resposta. Sobre destinos como o Fortaleza, por exemplo, muitos brasileiros dispõem de muitas informações armazenadas em suas memórias. Contudo os consumidores de turismo geralmente dispõem de pouca informação sobre os destinos, sendo seus conhecimentos acerca destas localidades resgatados de forma relativamente fácil e homogênea quando solicitado. A maior parte das pessoas provavelmente não é capaz de associar um destino como Jericoacoara (CE) a muito mais do que meia dúzia de coisas. Em muitas situações reais de escolha, o destino simplesmente não era previamente conhecido pelo indivíduo. Esse é um caso comum em escolhas feitas nos balcões de atendimento das agências de viagens ou ao longo do curso de viagens de múltiplos destinos. Se o indivíduo acaba de ser apresentado a um novo destino, vendo algumas fotos e ouvindo algumas informações do agente de viagens, como esperar que ele saiba distinguir e expressar suas percepções e emoções acerca da perspectiva de uma viagem para lá?

Por fim, deve-se destacar que a utilidade de escalas diretas e indiretas também se difere em função do grau de heterogeneidade de acessibilidade das informações sobre o objeto em questão na mente do indivíduo. De acordo com Ajzen (2005), escalas indiretas são mais recomendadas quando existem motivos para acreditar que algumas informações são mais acessíveis do que outras 
na mente do indivíduo. Desta forma é possível evitar o viés de recuperação desigual de memórias por meio da maior exigência de elaboração mental por parte dos respondentes da pesquisa. Por outro lado, escalas diretas são recomendáveis quando não existem motivos para acreditar em significativas diferenças de acessibilidade de informações sobre o objeto na mente do indivíduo. Diferenças na acessibilidade das informações estão associadas tanto à dimensionalidade das relações entre indivíduo e objeto, quanto ao nível de conhecimento daquele sobre este. Relações mais multidimensionais favorecem a heterogeneidade na acessibilidade de informações. Por outro lado, níveis reduzidos de conhecimento sobre o objeto tendem a favorecer a acessibilidade homogênea das informações de memória.

Em suma, escalas diretas são especialmente recomendadas para a mensuração das atitudes relativas a destinos turísticos quando o grau de conhecimento sobre estes é pequeno. Nestes casos, o prejuízo da superficialidade das avaliações não é grande, já que não há lugar para avaliações mais profundas. Também não se fazem presentes os prejuízos da heterogeneidade na acessibilidade de informações na mente do indivíduo. Por outro lado, evitam-se arbitrariedades na construção da escala, ampliando-se a validade de construto. Ainda mais importante, a escala direta não exige avaliações sobre aspectos específicos e muitas vezes pouco conhecidos do indivíduo, evitando o risco de respostas inconsistentes ou até inexistentes.

\section{CONFIABILIDADE, VALIDADE E EXTENSÃO DE ESCALAS}

A atitude estimada por meio de uma escala direta $(X)$ nem sempre é exatamente igual ao verdadeiro valor da atitude $(T)$. A diferença entre ambos é chamada de erro aleatório (E). Desta forma,

$$
X=T+E
$$

Sob estas condições, a variância total da estimativa $\left({ }^{\sigma_{X}^{2}}\right)$ é igual à soma da variância do verdadeiro valor da atitude $\left({ }^{\sigma_{T}^{2}}\right)$ e do termo de erro aleatório $\left.{ }^{\sigma_{E}^{2}}\right)$, isto é $\sigma_{X}^{2}=\sigma_{T}^{2}+\sigma_{E}^{2}$ 


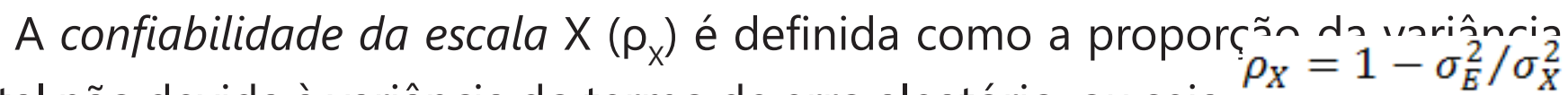
total não devida à variância do termo de erro aleatório, ou seja, (KROSNICK; JUDD; WITTENBRINK, 2005). A confiabilidade está associada à diferença de origem não sistemática entre aquilo que se pretendia medir e aquilo que é efetivamente mensurado. O resultado da escala pouco confiável é a obtenção de diferentes valores em mensurações repetidas.

No caso das atitudes, problemas de confiabilidade surgem sobretudo da natureza latente dessa variável. Cada indicador da atitude subjacente traz consigo, além da atitude em si, elementos aleatórios que distanciam seu valor daquilo que seria uma representação precisa da atitude. Essa diferença tende a ser ainda maior no caso de objetos pouco conhecidos, pois estes favorecem a inconsistência das respostas dos indivíduos.

Escalas de um único item, as quais coletam uma única informação e consideram esta como a estimativa da atitude subjacente, têm se provado bastante adequadas para a mensuração de atitudes em casos específicos (AJZEN, 2005). Contudo escalas desse tipo trazem consigo o perigo da falta de confiabilidade em sua expressão máxima. Em virtude disto, Fishbein e Ajzen (1975) defendem que escalas de único item não devem ser adotadas sem o suporte de evidências empíricas para o caso específico.

Uma alternativa frequentemente mais confiável são as escalas compostas por múltiplos itens. Neste tipo de escala os diferentes erros associados com cada item tendem a se cancelar mutuamente, fazendo com que a medida final da atitude seja relativamente pouco afetada (AJZEN, 2005). A vantagem da escala multi-itens se dá pelo processo de regressão à média das repetidas medidas obtidas junto a cada indivíduo. É importante destacar que esse processo pode ocorrer não apenas para diversas observações relativas a um mesmo indivíduo, mas também para observações referentes a diferentes indivíduos de uma mesma população. Em outras palavras, em estudos com vários indivíduos o perigo da baixa confiabilidade das medidas pode ser remediado por escalas de múltiplos itens ou por amostras maiores.

Dois itens são considerados paralelos se compartilham as mesmas causas e têm erros aleatórios do mesmo tamanho. A correlação entre dois itens paralelos 
$\left(^{r_{X_{1} X_{2}}}\right)$ é igual à confiabilidade de cada item, de forma que ${ }^{r_{X_{1} X_{2}}=\rho_{X_{1}}=\rho_{X_{2}}}$. Portanto a confiabilidade de uma estimativa da atitude pode ser mensurada a partir da correlação desta com outra estimativa paralela da mesma atitude. $\mathrm{Na}$ prática, essas medidas paralelas podem ser tanto diferentes itens de um mesmo questionário, quanto os mesmos itens reaplicados em diferentes momentos. Assumindo itens paralelos, a confiabilidade de uma escala de m itens $\left({ }^{\rho_{M}}\right)$ pode ser estimada por meio da fórmula de profecia de Spearman-Brown:

$$
\rho_{M}=\frac{m r_{i j}}{1+(m-1) r_{i j}}
$$

A generalização desta equação, que permite correlações variáveis de cada item com o verdadeiro valor da atitude, é o Alpha de Cronbach (CRONBACH, 1951):

$$
\alpha_{M}=\left(\frac{m}{m-1}\right)\left(1-\frac{\sum \sigma_{i}^{2}}{\sigma_{M}^{2}}\right)
$$

onde ${ }^{\sum \sigma_{i}^{2}}$ é a soma das variâncias de cada item e ${ }^{\sigma_{M}^{2}}$ é a variância da escala M. De acordo com essa perspectiva, itens que não apresentam alta correlação com o valor final da escala não são bons indicadores da atitude em questão, devendo ser eliminados da escala.

Enquanto a confiabilidade analisa aspectos internos da escala, questões externas são julgadas por critérios de validade. A validade divergente indica o poder que a escala tem de diferenciar o construto em questão de outras variáveis que não fazem parte do domínio de interesse (KROSNICK; JUDD; WITTENBRINK, 2005). Uma escala com validade divergente é aquela cujos valores não apresentam correlação com os valores de outros construtos teoricamente independentes. Contudo a preocupação com a validade divergente é apenas relevante no âmbito das escalas de atitude quando é adotada uma perspectiva indireta de mensuração. Nas escalas diretas o erro sistemático pode no máximo estar associado a questões linguísticas. Em outras palavras, pode-se questionar em que medida é possível expressar com palavras o verdadeiro conteúdo das 
atitudes. Mas, deixada a questão linguística de lado, deve-se assumir que escalas diretas não contêm erros sistemáticos, já que se referem diretamente à atitude.

Por sua vez, a validade de construto de uma escala está relacionada com as correlações não nulas teoricamente estabelecidas entre o construto em questão e outros (DEVELLIS, 2003). Tem validade de construto a escala cujas correlações esperadas em termos teóricos são empiricamente confirmadas.

A tentativa de criar diferentes itens indicativos da atitude em relação a um único objeto com frequência leva à abordagem indireta. Por outro lado, ao tangenciarem a atitude, em vez de atacá-la de frente, os itens indiretos podem ter mais variabilidade do que os itens diretos. Esse risco é ainda maior no caso de atitudes superficialmente definidas na mente do indivíduo. Portanto escalas indiretas de múltiplos itens nem sempre serão mais confiáveis. Se a variabilidade dos itens for muito grande, o resultado pode ser uma escala menos confiável do que uma escala direta. Naturalmente esse não é o caso mais comum, mas não pode ser descartado a priori.

Além disso, as escalas indiretas correm mais riscos de apresentarem validade reduzida. Novamente o problema advém do fato de que essas escalas tangenciam o objeto no lugar de abordá-lo abertamente. Corre-se aí o risco de incluir na escala itens que de alguma forma tangenciam também outros construtos. Este efeito parece ser mais provável no caso de objetos que despertam reações complexas e ensejam maior arbitrariedade na seleção dos itens da escala, como é o caso dos destinos turísticos.

A última qualidade das escalas a ser destacada nesta seção é a extensão. Escalas com muitos itens exigem maior esforço de levantamento, implicando mais tempo e maiores custos de pesquisa. Além disso, quanto maior o número de itens de uma escala, maior também é a dedicação exigida do respondente. De maneira geral, quanto maior o questionário, menor é a taxa de resposta (DILLMAN; SINCLAIR; CLARK, 1993; HEBERLEIN; BAUMGARTNER, 1978). Além disso, questionários maiores também prejudicam a qualidade das respostas obtidas (BURCHELL; MARSH, 1992; GALESIC; BOSNJAK, 2009). Logo, a seleção do número de itens de uma escala deve levar em conta também a economicidade e a qualidade das respostas obtidas. O menor esforço por parte do pesquisador e do pesquisado contribui para a preferência por escalas com menor número de itens. 


\section{ESCALAS DE ATITUDES SOBRE DESTINOS TURÍSTICOS}

A lista de escalas disponíveis na literatura para a mensuração das atitudes relativas a destinos turísticos não é grande. Um eCrompton (1990) desenvolveram um estudo pioneiro neste campo, criando uma escala indireta de atitudes em relação ao destino composta por uma série de 20 itens relacionados às percepções do indivíduo sobre essa localidade. Esse conjunto de itens foi selecionado por meio de uma pesquisa empírica com estudantes a partir de um conjunto inicial de 40 itens. Cada item foi avaliado pelos respondentes sob duas perspectivas distintas. Primeiramente, os respondentes avaliaram a posição do destino em uma escala de Likert de cinco pontos. Na sequência, cada item foi avaliado em uma escala de Likert de onze pontos para indicar se aquele aspecto deveria ser considerado como um facilitador ou um inibidor da viagem ao destino. Ambos os valores foram multiplicados para cada item e o resultado dessas multiplicações foi somado para compor uma estimativa final da atitude do indivíduo em relação ao destino.

Mohsin (2005) estudou as atitudes de turistas malaios acerca do território Norte da Austrália como destino turístico. Para tanto, o autor desenvolveu uma escala indireta com 62 itens do tipo Likert com sete pontos relativos às percepções dos indivíduos. Uma análise fatorial exploratória foi aplicada sobre um conjunto de observações coletadas, a fim de identificar diferentes dimensões das atitudes. O resultado apontou a existência de doze dimensões significativas, oferecendo indícios de que a atitude global em relação ao destino pode não ser composta por percepções consistentes acerca de diferentes características deste.

Para além das percepções, Lee (2009) mensurou atitudes relativas a destinos turísticos em Taiwan por meio de uma escala indireta composta por onze itens que tratavam de diferentes aspectos. Cada item era composto por uma escala de Likert de sete pontos. Análise fatorial exploratória e análise confirmatória foram utilizadas para examinar os dados coletados. Os onze itens foram agrupados em três fatores, refletindo percepções, emoções e intenções dos indivíduos. $O$ estudo mostrou que a atitude global é um dos determinantes da satisfação e do comportamento futuro dos indivíduos. 
Phillips e Jang (2008) analisaram a formação das atitudes, examinando o papel das percepções e das emoções sobre a atitude global. O estudo foi baseado nas atitudes de turistas potenciais do meio oeste norte-americano acerca de Nova Iorque como destino turístico. Os autores utilizaram uma escala direta composta por três itens de diferencial semântico com sete pontos cada. Em cada item os respondentes indicaram se uma visita a Nova Iorque na viagem de férias de verão seria bom ou ruim, agradável ou desagradável, valiosa ou desvaliosa. Os dados foram analisados por meio de análise fatorial exploratória e confirmatória. Os resultados apontaram que as percepções têm efeito apenas indireto sobre as atitudes, sendo mediadas pelas emoções.

Sparks e Pan (2009) utilizaram uma escala direta para mensurar as atitudes de turistas chineses em relação ao destino turístico Austrália. Os autores utilizaram uma escala direta composta por seis itens de diferencial semântico com sete pontos, questionando se "considerando todas as questões, fazer uma viagem de férias para a Austrália nos próximos 12 meses seria..." aprazível ou desaprazível, bom ou ruim, divertido ou ridículo, agradável ou desagradável, favorável ou desfavorável, atraente ou abominável. Baseando-se na Teoria do Comportamento Planejado (AJZEN, 1991), o estudo examinou a influência das atitudes sobre as intenções dos indivíduos. Contrariamente ao esperado, os resultados apontaram que as intenções de viagem são determinadas pelas normas subjetivas e pelo controle percebido. Contudo, não foram encontradas evidências de que as intenções sejam influenciadas pelas atitudes. Os autores sugerem que esse resultado pode representar uma característica particular do público chinês.

Hsu, Cai e Li (2010) também examinaram os antecedentes das atitudes se atendo aos efeitos das expectativas e das motivações sobre essa variável dependente. As atitudes foram mensuradas por meio de uma escala direta composta por seis itens do tipo Likert de sete pontos, avaliando se "A partir de todo seu conhecimento, você acredita que uma visita a Hong Kong seria..." satisfatória, agradável, aprazível, fascinante e que vale a pena. Os resultados foram analisados por meio de análise fatorial e confirmaram que ambas as variáveis explicativas têm efeito direto sobre as atitudes. 
Kim e Chen (2010) e Kim, Chen e Hwang (2011) estudaram o grau de consciência dos indivíduos sobre suas próprias atitudes relativas a destinos turísticos. Para tanto, os autores compararam medidas explícitas e implícitas das atitudes de norte-americanos em relação aos destinos turísticos Reino Unido e China. A mensuração explícita das atitudes foi realizada com base em uma escala de quatro itens do tipo Likert com cinco pontos cada. Os itens utilizados foram: $O$ país $X$ é um bom destino para se viajar, o país $X$ é um destino de viagem único, eu gostaria de viajar para o país $X$, eu gosto da ideia e viajar para o país $X$. Os resultados apontaram que as medidas explícitas e implícitas são essencialmente consistentes entre si.

Morakabati, Fletcher e Prideaux (2012) utilizaram uma escala baseada apenas em intenções para mensurar as atitudes de turistas em relação a destinos de alto risco. A escala direta, composta por dois itens do tipo Likert com cinco pontos cada, avaliou o grau de concordância dos sujeitos com as declarações "definitivamente não iria" e "definitivamente gostaria de visitar" relativas aos destinos em questão.

Jalilvand et al. (2012) avaliaram a influência das atitudes sobre as intenções relativas à seleção do destino de viagem, considerando concomitantemente os efeitos da imagem do destino e do 'boca a boca' eletrônico. O estudo analisou as intenções de retorno ao país de turistas internacionais em visita a Isfahan no Irã. A mensuração das atitudes foi feita por meio de uma escala direta composta por três itens de diferencial semântico. Os respondentes indicaram se, "como um destino turístico, o Irã é" muito bom ou muito ruim, sem nenhum valor ou muito valioso, muito agradável ou muito desagradável. Os resultados, estimados a partir de análise fatorial confirmatória, confirmaram que as atitudes influenciam positivamente as intenções de viagem. Este estudo foi replicado por Jalilvand, Ebrahimi e Samiei (2013) para destinos islâmicos em geral, utilizandose a mesma escala e obtendo-se resultados semelhantes.

Um estudo especialmente interessante para o contexto deste trabalho é aquele desenvolvido por Kim e Stepchenkova (2015). Esses autores avaliaram o impacto de fotografias feitas por turistas sul-coreanos e norte-americanos sobre as atitudes dos turistas daqueles países em relação ao destino turístico 
Rússia. As fotografias foram apresentadas aos sujeitos da pesquisa e avaliadas segundo critérios, como lotação dos espaços, limpeza, segurança, modernidade e outros. Na sequência, as atitudes dos sujeitos em relação ao destino foram mensuradas por meio de uma escala direta de item único: "baseado nesta foto, quão positiva é sua impressão da Rússia como destino?". Os autores afirmaram que as implicações teóricas da utilização de escalas de item único em comparação com escalas de múltiplos itens foram analisadas, mas que se chegou à conclusão de que a primeira alternativa era suficiente para o propósito da pesquisa, além de reduzir a exigência sobre o respondente. Os resultados foram analisados por meio de regressão e indicaram quais atributos das fotos têm maior influência sobre as atitudes dos turistas potenciais.

As escalas diretas constituem uma parcela significativa do total de estudos de atitudes relativas a destinos turísticos. Contudo o processo de desenvolvimento desses instrumentos de mensuração foi apenas superficialmente discutido. A maioria dos estudos apresentados tem foco em questões que vão além das próprias escalas, destinando pouca atenção ao desenvolvimento destas. As escalas não foram adequadamente justificadas em termos de validade, confiabilidade e economicidade. Logo, estudos futuros que necessitem utilizar escalas diretas de atitudes relativas a destinos turísticos não contam atualmente com apoio teórico e empírico sólido. Desta forma, a seção a seguir desenvolve cuidadosamente uma escala dessa natureza, discutindo cada passo do processo de desenvolvimento, a fim de compor um instrumento transparente e eficiente.

\section{DESENVOLVIMENTO DA ESCALA DE ATITUDE EM RELAÇÃO A DESTINOS TURÍSTICOS}

O desenvolvimento de uma escala direta para mensuração das atitudes relativas a destinos turísticos foi realizado com base no processo padrão de oito etapas sugerido por DeVellis (2003). Cada etapa tem diferentes objetivos e é composta por diferentes procedimentos metodológicos, conforme descrito a seguir. 


\section{DELIMITAÇÃO DA VARIÁVEL}

A primeira etapa sugerida por DeVellis (2003) é a delimitação da variável a ser mensurada. No caso das atitudes, Ajzen (2005) sugere que seja definido claramente qual o objeto sobre o qual a atitude se refere e quais as reações do indivíduo incluídas no domínio da atitude avaliada. No presente trabalho o objeto de interesse é o destino turístico. Como tal, as reações incluídas no domínio da atitude são aquelas relativas às viagens turísticas para essa localidade. Uma vez que as atitudes são estudadas como antecedentes das escolhas do consumidor, o foco da análise será direcionado para as viagens em que o destino é mais claramente selecionado pelo indivíduo entre diferentes alternativas. Desta forma, a presente análise se concentrará nas viagens de lazer, alternativa que mais se associa a processos de livre escolha, distanciando-se das obrigações, das facilidades e dos outros fatores determinantes externos ao indivíduo.

\section{ELABORAÇÃO DA LISTA INICIAL DE ITENS}

A segunda etapa consiste na elaboração de uma lista preliminar de itens que poderão vir a compor a escala. A definição da atitude a ser mensurada deve guiar esta etapa, a fim de que sejam elaborados itens capazes de captar a essência desta variável sob diferentes perspectivas. A elaboração dessa lista pode se apoiar tanto no exame cuidadoso da definição quanto em estudos empíricos realizados anteriormente. É desejável que o conjunto de itens seja grande. Certa redundância entre os itens deve ser considerada como uma qualidade do conjunto.

A redação dos itens é composta de duas partes, o enunciado e a resposta (DOLNICAR, 2013). A primeira parte apresenta o elemento a ser avaliado e a segunda estabelece quais são as alternativas de avaliação. Uma vez que este trabalho se propõe a mensurar as atitudes relativas a destinos turísticos por meio de uma escala direta, o enunciado estabelecido para os itens desta pesquisa é simples e objetivo: Como você avalia esta localidade como destino para viagens de lazer? 


\section{DEFINIÇÃO DO FORMATO DE MENSURAÇÃO}

Existem diferentes formatos de mensuração, incluindo escalas de Thurstone, Guttman, Likert e de diferencial semântico. Estas duas últimas são as mais comumente utilizadas para a mensuração de atitudes. Krosnick, Judd e Wittenbrink (2005) defendem o uso das escalas de diferencial semântico por considerá-las mais simples e de fácil aplicação. Friborg, Martinussen e Rosenvinge (2006) comparam estas às escalas de Likert em um estudo empírico sobre a mensuração de construtos psicológicos e também concluem que a escala de diferencial semântico apresenta vantagens em comparação com a escala de Likert. Desta forma, optou-se por elaborar itens no formato de diferencial semântico.

Com respeito ao número de pontos de cada item, diferentes práticas têm sido adotadas na literatura específica. De acordo com Krosnick, Judd e Wittenbrink (2005), o número de pontos para cada item deve ser condizente com a precisão das análises passíveis de elaboração pelos indivíduos sobre o tema. Se os indivíduos diferenciam não mais do que sete categorias, adicionar mais pontos à escala não irá oferecer nenhuma informação adicional ao pesquisador. Lissitz e Green (1975) mostram que escalas com menos de cinco pontos apresentam confiabilidade reduzida, mas que a partir desse tamanho o aumento no número de pontos não amplia a confiabilidade da escala. A partir de uma ampla revisão, Krosnick, Judd e Wittenbrink (2005) indicam que as escalas com um número moderado de pontos são as mais indicadas tanto em relação à confiabilidade quanto à validade. Assim sendo, optou-se por elaborar itens com sete pontos, seguindo o formato original da escala de diferencial semântico.

Uma vez que todos os itens da lista inicial têm o mesmo enunciado, o número de itens é dado pelas diferentes respostas. Cada item é definido por um par de termos que baliza os extremos da escala de sete pontos. A elaboração da lista de pares de termos nesta pesquisa teve como ponto de partida a compilação feita por Bruner II (2009) de escalas de atitudes relativas ao objeto e relativas ao produto. Tal compilação foi realizada a partir da revisão de mais de 200 trabalhos publicados. A lista elaborada por Bruner II contém 53 pares de termos em 
língua inglesa. Uma das dificuldades enfrentadas pela tradução desses termos decorre do fato de que alguns deles correspondem a mais de uma palavra na língua portuguesa, assim como em outros casos dois ou mais termos do inglês correspondem a apenas uma palavra no português. O termo enjoyable, por exemplo, pode ser traduzido como agradável ou aprazível. Por outro lado, agradável também é uma tradução viável do termo inglês pleasant.

A busca por termos opostos na língua portuguesa também trouxe desafios. O antônimo de mesma raiz etimológica não existe para alguns termos, como é o caso do termo positivo, o qual é contraposto pelo termo negativo. Além disso, para alguns termos existe mais de um antônimo viável. O termo adorável, por exemplo, pode ser contraposto por termos como abominável, odioso e aborrível. Outra opção é contrapor termos que não tem antônimo de mesma raiz etimológica com termos compostos por palavras que invertem o sentido. Nesta perspectiva, atraente pode ser contraposto por não atraente.

Por fim, a partir da lista de termos traduzida da compilação de Bruner II, foi realizada uma tentativa de ampliação a partir da busca por termos parecidos no dicionário Houaiss de sinônimos (INSTITUTO ANTÔNIO HOUAISS, 2003). Desta forma, elaborou-se um conjunto de 62 pares de termos para compor as respostas dos itens de diferencial semântico.

\section{REVISÃO DA LISTA INICIAL DE ITENS}

A lista inicial de itens deve ser revisada, a fim de excluir elementos que não se ajustem à atitude definida como objeto da mensuração. Neste trabalho foram excluídos os itens que:

- Fazem referência a características específicas do objeto, não sendo apropriados para o uso em escalas diretas de atitudes (por exemplo: barato/ caro).

- Fazem referência às intenções dos indivíduos (por exemplo: visitaria/não visitaria). 
- Não se estendem por todo contínuo das avaliações possíveis, começando ou terminando em níveis intermediários, em vez de extremos (por exemplo: satisfatório/insatisfatório, já que um destino turístico pode ser mais que satisfatório).

- São compostos por termos pouco utilizados e com menor chance de serem facilmente compreendidos pelos respondentes (por exemplo: aprazível/ desaprazível).

- São compostos por termos ambíguos, podendo ser interpretados pelo respondente em outro sentido que não aquele de interesse da pesquisa (por exemplo: consistente/inconsistente).

- São excessivamente redundantes em comparação com outros itens já incluídos (por exemplo: bom/ruim em comparação com excelente/péssimo).

A partir destes critérios, a lista inicial de itens foi reduzida a seis itens:

1. Adorável / Detestável

2. Agradável / Desagradável

3. Atraente / Não atraente

4. Desejável / Indesejável

5. Excelente / Péssimo

6. Positivo / Negativo

\section{INCLUSÃO DE ITENS DE VALIDAÇÃO}

A teoria afirma que atitudes e intenções relativas a um objeto devem ser positivamente correlacionadas. Desta forma, a validade de construto da escala de atitude em relação a destinos turísticos pode ser avaliada a partir da análise da correlação desta com as intenções de viagem do indivíduo. Assim sendo, procedeu-se à mensuração das intenções relativas ao destino turístico a partir 
de uma escala de três itens de diferencial semântico de sete pontos inspirada nas escalas utilizadas por Jalilvand et al. (2012) e Kim e Stepchenkova (2015) e apresentada a seguir:

- Eu gostaria de visitar [o destino] (concordo/discordo)

- Eu visitaria [o destino] antes de qualquer outro (concordo/discordo)

- Acredito que vou visitar [o destino] no futuro (concordo/discordo)

\section{APLICAÇÃO DA ESCALA A UMA AMOSTRA DE DESENVOLVIMENTO}

O teste da escala com vistas a seu refinamento e validação deve ser feito a partir da aplicação do instrumento de coleta de dados a uma amostra de indivíduos. Grande parte dos estudos desse tipo tem utilizado amostras de indivíduos que fazem parte de grupos específicos, como estudantes, por exemplo. DeVellis (2003) defende que esse tipo de amostra geralmente não apresenta problemas para o desenvolvimento de escalas, ainda que os valores obtidos não sejam necessariamente representativos de uma população mais abrangente. Isto ocorre porque, ainda que o público específico pesquisado tenha atitudes diferentes da população em geral, as relações existentes entre os itens e a atitude, bem como entre a atitude e outras variáveis, tendem a ser as mesmas para todos os grupos de indivíduos. Em outras palavras, a amostra não seria adequada caso fosse constituída por indivíduos com características mentais qualitativamente distintas, e não simplesmente com distinções quantitativas.

Neste trabalho o instrumento de pesquisa foi aplicado entre abril e maio de 2015 a uma amostra de 103 alunos de graduação das três áreas do conhecimento de uma instituição pública do estado de São Paulo. A seleção de alunos de graduação também se justifica pela capacidade cognitiva relativamente elevada exigida pelo instrumento de coleta. Os questionários de autorresposta foram aplicados com auxílio de tablets, o que facilitou a apresentação de fotos dos destinos e a tabulação dos dados. 
As escalas desenvolvidas foram aplicadas três vezes a cada indivíduo, permitindo a mensuração das atitudes relativas a destinos turísticos em três situações, cada uma se referindo a um nível distinto de heterogeneidade da acessibilidade de informações na memória do indivíduo. Na primeira mensuração apenas o nome de um destino muito conhecido (Fortaleza) é mencionado, sendo todas as demais informações sobre este resgatadas diretamente da memória do indivíduo. Neste caso pode-se prever certo grau de heterogeneidade na acessibilidade das informações resgatadas. $\mathrm{Na}$ segunda mensuração o nome de um destino pouco conhecido (Jericoacoara) é mencionado e um conjunto de dez fotos é apresentado ao respondente. Desta forma, algumas informações são prontamente apresentadas ao indivíduo, enquanto outras são eventualmente resgatadas de sua memória, resultando em uma situação intermediária de heterogeneidade de acessibilidade de informações. Por fim, na terceira mensuração um destino fictício é apresentado ao indivíduo por meio de um conjunto de dez fotos. Como o nome do destino não é mencionado, apenas as informações apresentadas ao indivíduo encontram-se diretamente disponíveis, implicando uma situação de baixa heterogeneidade de acessibilidade de informações.

\section{AVALIAÇÃO DOS ITENS}

A contribuição de cada um dos seis itens da escala para a mensuração da atitude relativa aos destinos turísticos foi inicialmente avaliada segundo a estatística Alpha de Cronbach. Os valores do Alpha de Cronbach em cada situação para a escala completa e para escalas que excluem um dos itens originais são apresentados na Tabela 1 . Os resultados indicaram excelente grau de confiabilidade (HAIR et al., 2010). Todos os seis itens apresentam contribuição para a escala, já que nas três situações consideradas o valor do Alpha seria reduzido caso qualquer um dos seis itens fosse omitido. Nota-se ainda que o valor do Alpha cresce conforme diminui o grau de conhecimento do indivíduo sobre o destino avaliado, indicando maior confiabilidade da escala quando aplicada a destinos menos conhecidos. 
Tabela 1: Alpha de Cronbach da escala completa e escalas reduzido

\begin{tabular}{|c|c|c|c|c|}
\hline & Escala & $\begin{array}{l}\text { Destino muito } \\
\text { conhecido }\end{array}$ & $\begin{array}{c}\text { Destino pouco } \\
\text { conhecido }\end{array}$ & $\begin{array}{c}\text { Destino } \\
\text { hipotético }\end{array}$ \\
\hline \multirow{7}{*}{ 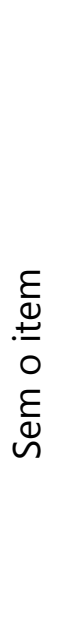 } & Completa & 0,929 & 0,950 & 0,966 \\
\hline & Adorável / Detestável & 0,914 & 0,938 & 0,961 \\
\hline & Agradável / Desagradável & 0,902 & 0,940 & 0,959 \\
\hline & Atraente / Não atraente & 0,914 & 0,947 & 0,958 \\
\hline & Desejável / Indesejável & 0,924 & 0,936 & 0,958 \\
\hline & Excelente / Péssimo & 0,920 & 0,940 & 0,963 \\
\hline & Positivo / Negativo & 0,919 & 0,943 & 0,960 \\
\hline
\end{tabular}

\section{OTIMIZAÇÃO DA ESCALA}

A otimização da escala pode ser feita a partir da solução do dilema entre extensão e confiabilidade. De acordo com DeVellis (2003)

Geralmente, escalas mais curtas são boas porque sobrecarregam menos os respondentes. Escalas mais longas, por outro lado, são boas porque tendem a ser mais confiáveis. Obviamente, maximizar um desses aspectos reduz o outro. Portanto, o desenvolvedor de escalas deve refletir sobre o equilíbrio ideal entre brevidade e confiabilidade. (DEVELLIS, 2003, p. 97, tradução nossa).

Ainda que nas três situações estudadas o valor do Alpha de Cronbach para a escala completa de seis itens seja bastante alto, a contribuição adicional de cada item da escala é relativamente pequena. Os itens mais representativos apresentam correlações com o escore total acima de 0,89 , conforme apresentado na Tabela 2. Isso indica que mesmo escalas de item único são altamente confiáveis. Ademais, nota-se que os níveis de correlação entre cada item e o escore total são maiores quanto menor for o conhecimento do indivíduo sobre o destino em questão. Isto indica que quanto menor o grau de conhecimento sobre o objeto, mais confiável é a escala de item único. 
Tabela 2: Correlação entre cada item da escala e o escore total

\begin{tabular}{cccc}
\hline Item & $\begin{array}{c}\text { Destino muito } \\
\text { conhecido }\end{array}$ & $\begin{array}{c}\text { Destino pouco } \\
\text { conhecido }\end{array}$ & Destino hipotético \\
\hline Adorável / Detestável & 0,803 & 0,873 & 0,884 \\
Agradável / Desagradável & 0,892 & 0,871 & 0,897 \\
Atraente / Não atraente & 0,806 & 0,785 & 0,910 \\
Desejável / Indesejável & 0,736 & 0,890 & 0,913 \\
Excelente / Péssimo & 0,759 & 0,854 & 0,858 \\
Positivo / Negativo & 0,766 & 0,827 & 0,888 \\
\hline
\end{tabular}

O prejuízo resultante da redução do número de itens da escala foi mais detalhadamente analisado a partir da comparação de dois modelos de equações estruturais. O primeiro modelo representa o caso completo no qual a atitude e a intenção são consideradas como variáveis latentes construtivas dos itens de cada escala e a atitude é tida como antecedente da intenção, conforme apresentado na Figura 1. Esse modelo foi rodado para cada situação de análise (destino muito conhecido, pouco conhecido ou hipotético). Os três modelos apresentaram bom nível de ajuste aos dados, sendo considerados significantes pelo Critério Consistente de Informação de Akaike - CAIC (ANDERSON; BURNHAM; WHITE, 1998). Todos os coeficientes também foram estimados como significantes para todas as relações consideradas, incluindo a relação entre as variáveis latentes atitude e intenção. Os coeficientes padronizados são apresentados na Tabela 3. 
Figura 1: Modelo completo

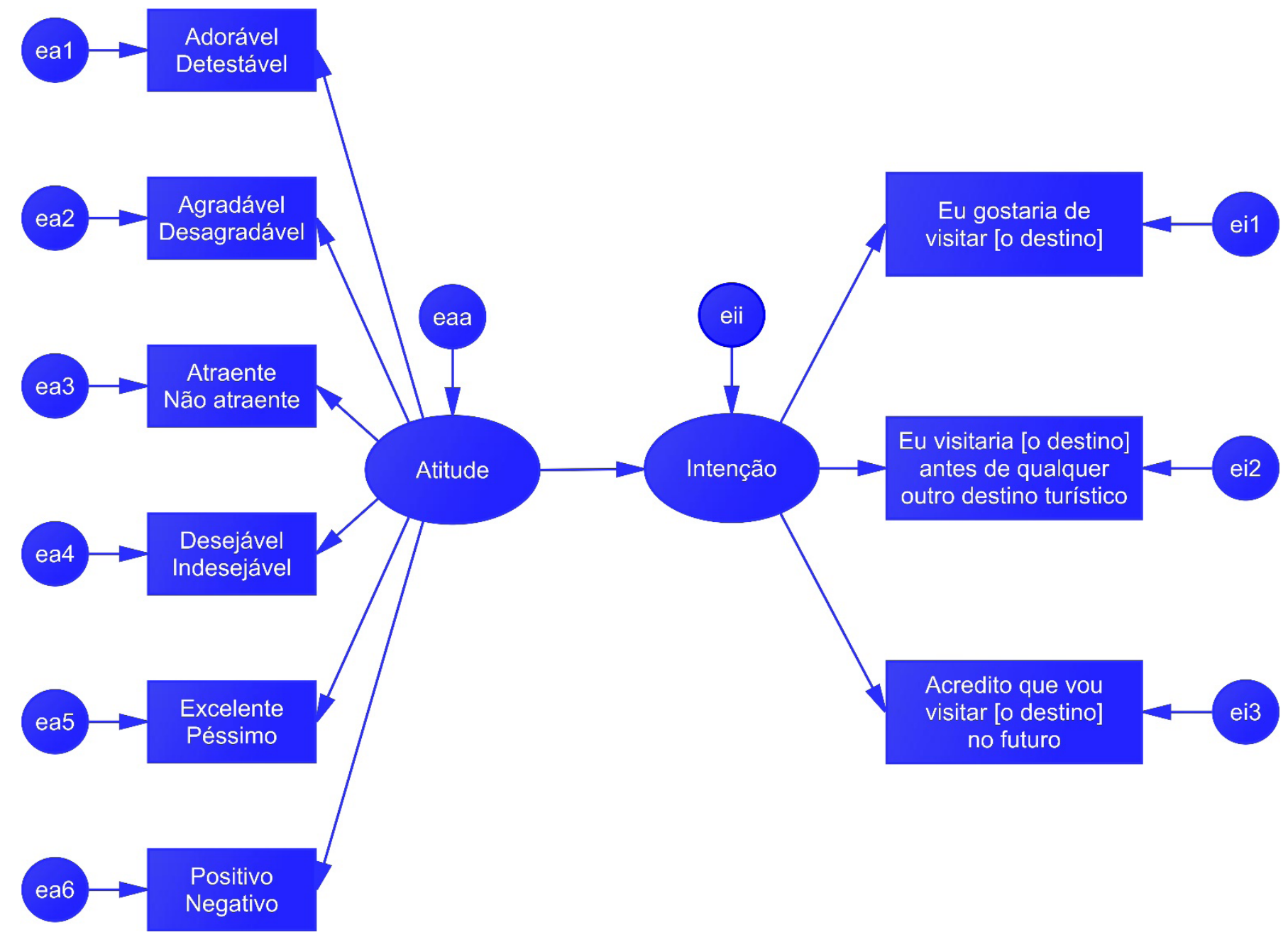

Tabela 3: Coeficientes padronizados do modelo completo

\begin{tabular}{cccc}
\hline Relação & \multicolumn{3}{c}{ Situação } \\
\cline { 2 - 4 } & $\begin{array}{c}\text { Destino muito } \\
\text { conhecido }\end{array}$ & $\begin{array}{c}\text { Destino pouco } \\
\text { conhecido }\end{array}$ & $\begin{array}{c}\text { Destino } \\
\text { hipotético }\end{array}$ \\
\hline Atitude $\rightarrow$ Adorável / Detestável & 0,865 & 0,905 & 0,907 \\
Atitude $\rightarrow$ Agradável / Desagradável & 0,95 & 0,878 & 0,913 \\
Atitude $\rightarrow$ Atraente / Não atraente & 0,844 & 0,8 & 0,932 \\
Atitude $\rightarrow$ Desejável / Indesejável & 0,769 & 0,93 & 0,931 \\
Atitude $\rightarrow$ Excelente / Péssimo & 0,768 & 0,876 & 0,875 \\
Atitude $\rightarrow$ Positivo / Negativo & 0,766 & 0,854 & 0,904 \\
Intenção $\rightarrow$ Eu gostaria de visitar [o destino] & 0,786 & 0,924 & 0,901 \\
Intenção $\rightarrow$ Eu visitaria [o destino] antes de qualquer & 0,522 & 0,508 & 0,602 \\
outro & & & 0,752 \\
Intenção $\rightarrow$ Acredito que vou visitar [o destino] no & 0,79 & 0,794 & 0,809 \\
futuro & 0,814 & 0,734 & \\
\hline
\end{tabular}


O segundo modelo utiliza apenas um item como indicador da atitude, constituindo uma escala de item único. Nesse modelo, as variáveis latentes atitude e intenção desaparecem, dando lugar apenas ao item relativo à atitude, cujo valor foi efetivamente observado, conforme apresentado na Figura 2. Cada um dos seis itens da escala completa foi testado nessa posição para cada uma das três situações de análise, totalizando 18 modelos estimados. Os resultados desses modelos foram comparados aos resultados dos três modelos completos estimados anteriormente por meio do Critério Consistente de Informação de Akaike - CAIC (ANDERSON; BURNHAM; WHITE, 1998). Em todos os casos o modelo reduzido se mostrou melhor do que o modelo completo. No pior caso, o modelo reduzido apresentou um CAIC 25,1\% inferior ao modelo completo da mesma situação. No caso mais destacado, o modelo reduzido chegou a apresentar um CAIC 52,8\% menor que o modelo completo. Os valores do CAIC estimados para todos os modelos são apresentados na Tabela 4.

Figura 2: Modelo reduzido

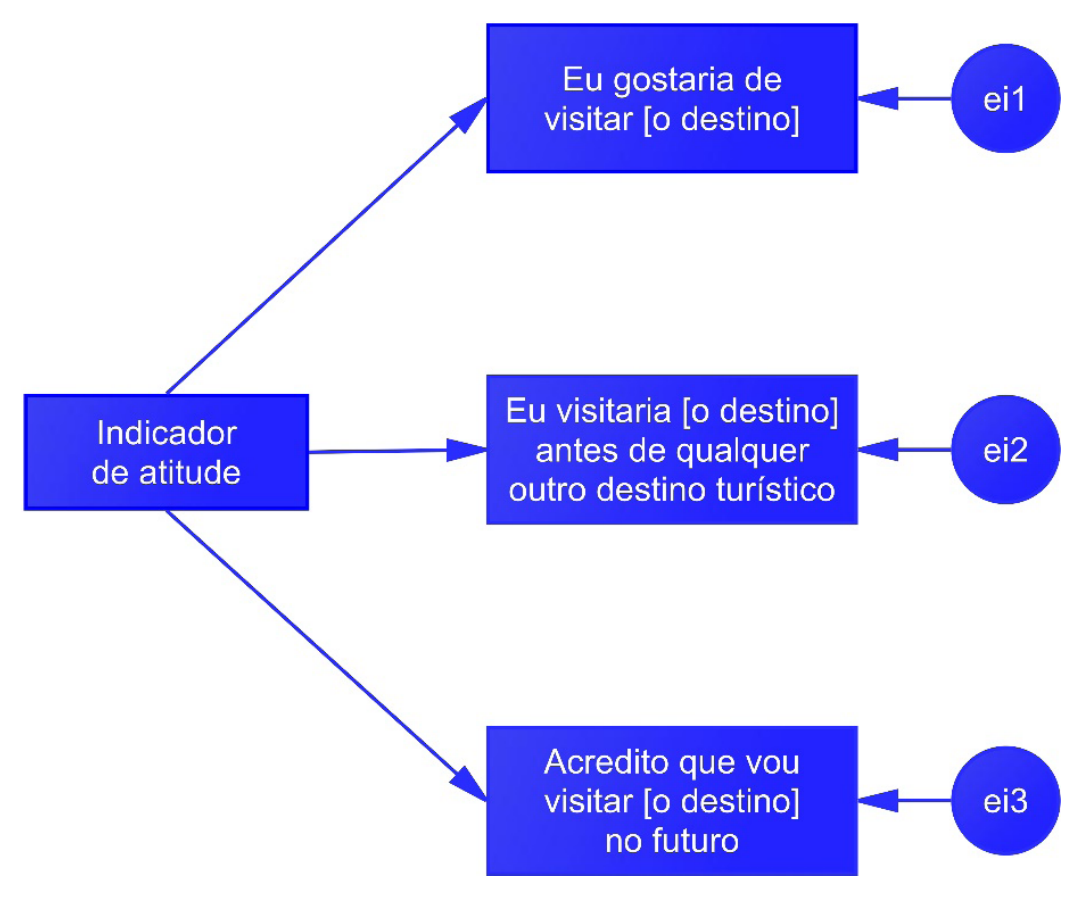


Tabela 4: Critério Consistente de Informação de Akaike para o modelo completo e modelos reduzidos

\begin{tabular}{|c|c|c|c|c|}
\hline & Modelo & $\begin{array}{l}\text { Destino muito } \\
\text { conhecido }\end{array}$ & $\begin{array}{l}\text { Destino pouco } \\
\text { conhecido }\end{array}$ & Destino hipotético \\
\hline \multirow{7}{*}{$\begin{array}{l}\frac{0}{0} \\
\frac{0}{N} \\
\frac{7}{0} \\
\mathbb{Q}\end{array}$} & Completo & 138,9 & 194,3 & 167,0 \\
\hline & Adorável / Detestável & 75,4 & 110,9 & 121,6 \\
\hline & Agradável / Desagradável & 63,4 & 124,1 & 117,8 \\
\hline & Atraente / Não atraente & 80,8 & 124,7 & 103,9 \\
\hline & Desejável / Indesejável & 90,0 & 99,9 & 107,5 \\
\hline & Excelente / Péssimo & 89,8 & 114,4 & 110,2 \\
\hline & Positivo / Negativo & 87,2 & 106,1 & 99,6 \\
\hline
\end{tabular}

O desempenho de cada um dos seis itens como indicador da atitude, e consequentemente como variável explicativa dos itens relacionados à intenção, é relativamente homogêneo. Nas três situações, a diferença entre os itens com melhor e pior desempenho é menor do que a diferença entre o item com pior desempenho e modelo completo. Logo, escalas de único item são melhores do que escalas multi-itens, mesmo que o item com pior desempenho seja selecionado. Por mais que as escalas multi-itens sejam ligeiramente mais confiáveis, como aponta o Alpha de Cronbach, esse ganho não compensa a perda de graus de liberdade na explicação das intenções dos indivíduos.

O melhor item na situação do destino muito conhecido foi aquele cujos extremos eram identificados pelos termos agradável e desagradável. Na situação intermediária do destino pouco conhecido, o item com melhor desempenho foi desejável/indesejável. Por fim, na situação do destino hipotético o melhor item foi o último: positivo/negativo.

A escala de único item se mostra comparativamente melhor na situação do destino muito conhecido. Isso indica que a maior solidez das atitudes relativas a destinos desse tipo faz com que o grau de confiabilidade de cada item da escala de atitude seja ampliado. No entanto, isso não quer dizer que a escala de único item seja inadequada para os destinos menos conhecidos. Na verdade, esse tipo de escala se mostrou superior à escala multi-itens em todos os casos, apenas apresentando diferentes níveis de superioridade. 
Apesar da grande importância das atitudes para explicação e previsão do comportamento dos consumidores, a mensuração desse construto é uma tarefa relativamente delicada em razão de sua natureza latente. Mensurar as atitudes relativas a um destino turístico pode contribuir para o desenvolvimento de muitas análises, incluindo aquelas em que as atitudes são o objeto central e outras cujas atitudes são apenas parte de raciocínios e objetivos mais complexos. Contudo, antes deste trabalho, a mensuração desse tipo de atitude ainda não contava com nenhum instrumento suportado por evidências empíricas que adotasse uma perspectiva direta.

Escalas diretas são mais simples e objetivas, evitando discussões sobre a validade da escala. Além disso, argumenta-se que as escalas diretas se prestam principalmente à análise de atitudes relativas a destinos turísticos pouco conhecidos, já que nestes casos o indivíduo teria poucas condições de avaliar diferentes aspectos desse objeto. A preocupação com a confiabilidade da escala que usualmente leva à adoção de escalas multi-itens, e consequentemente indiretas, não se mostrou relevante neste estudo. As escalas diretas de único item aqui desenvolvidas apresentaram alto grau de confiabilidade. As escalas também apresentaram alto nível de validade, constituindo explicações válidas da intenção de viagem dos indivíduos.

O processo de otimização do tamanho da escala indicou que um único item é suficiente para a realização de inferências confiáveis e válidas sobre a atitude relativa a destinos turísticos. Escalas multi-itens se mostraram ligeiramente mais confiáveis, mas esse ganho não compensou a redução dos graus de liberdade na explicação das intenções dos indivíduos. Além do mais, deve-se lembrar de que escalas com menor número de itens favorecem o aumento da taxa de resposta dos indivíduos abordados pela pesquisa, a melhoria da qualidade das respostas fornecidas pelos entrevistados e a economia de recursos na realização da pesquisa.

Portanto estes resultados levam à recomendação da utilização de escalas diretas de único item para a mensuração da atitude relativa a destinos 
turísticos. Essa recomendação vale para qualquer nível de heterogeneidade da acessibilidade de informações na memória do indivíduo, ainda que seja mais forte para situações de grande heterogeneidade. O melhor conteúdo do item utilizado como indicador da atitude não se mostrou o mesmo para as três situações estudadas. Contudo os resultados apontaram também que todos os seis itens estudados constituem bons indicadores.

\section{REFERÊNCIAS}

\section{AJZEN, I. The theory of planned behavior. Organizational Behavior and Human Decision} Processes, v. 50, n. 2, p. 179-211, 1991.

AJZEN, I. Attitudes, Personality and Behavior. 2. ed. Maidenhead: Open University Press, 2005.

ALBARRACÍN, D. et al. Attitudes: introduction and scope. In: ALBARRACÍN, D.; JOHNSON, B. T.; ZANNA, M. P. (Org.). The Handbook of Attitudes. Mahwah (EUA): Lawrence Erlbaum Associates, 2005. p. 3-20.

ANDERSON, D.; BURNHAM, K.; WHITE, G. Comparison of Akaike Information Criterion and Consistent Akaike Information Criterion for model selection and statistical inference from capture-recapture studies. Journal of Applied Statistics, v. 25, n. 2, p. 263-282, 1998.

BRUNER II, G. C. Marketing Scales Handbook: a compilation of multi-item measures for consumer behavior \& advertising research. Carbondale: GCBII, 2009.

BURCHELL, B.; MARSH, C. The effect of questionnaire length on survey response. Quality and Quantity, v. 26, n. 3, p. 233-244, 1992.

CRONBACH, L. J. Coefficient alpha and the internal structure of tests. Psychometrika, v. 16, n. 3, p. 297-334, 1951.

DEVELLIS, R. F. Scale Development: theory and applications: Applied Social Research Methods. 2. ed. Thousand Oaks: Sage Publications, 2003.

DILLMAN, D. A.; SINCLAIR, M. D.; CLARK, J. R. Effects of questionnaire length, respondentfriendly design, and a difficult question on response rates for occupant-addressed census mail surveys. Public Opinion Quarterly, v. 57, n. 3, p. 289-304, 1993.

DOLNICAR, S. Asking good survey questions. Journal of Travel Research, v. 52, n. 5, p. 551-574, 2013. 
EAGLY, A. H.; CHAIKEN, S. The Psychology of Attitudes. Orlando: Harcourt Brace Jovanovich College Publishers, 1993.

FARH, J. L.; CANNELLA, A. A.; LEE, C. Approaches to scale development in Chinese management research. Management and Organization Review, v. 2, n. 3, p. 301-318, 2006.

FISHBEIN, M.; AJZEN, I. Belief, Attitude, Intention, and Behavior: an introduction to theory and research. Reading: Addison-Wesley Publishing Company, 1975.

FRIBORG, O.; MARTINUSSEN, M.; ROSENVINGE, J. H. Likert-based vs. semantic differentialbased scorings of positive psychological constructs: A psychometric comparison of two versions of a scale measuring resilience. Personality and Individual Differences, v. 40, n. 5, p. 873-884, 2006.

GALESIC, M.; BOSNJAK, M. Effects of questionnaire length on participation and indicators of response quality in a web survey. Public Opinion Quarterly, v. 73, n. 2, p. 349-360, 2009.

HAIR, J. F. et al. Multivariate Data Analysis. Upper Saddle River: Prentice Hall, 2010.

HEBERLEIN, T. A.; BAUMGARTNER, R. Factors affecting response rates to mailed questionnaires: a quantitative analysis of the published literature. American Sociological Review, v. 43, n. 4, p. 447-462, 1978.

HSU, C. H. C.; CAI, L. A.; LI, M. Expectation, motivation, and attitude: a tourist behavioral model. Journal of Travel Research, v. 49, n. 3, p. 282-296, 2010.

INSTITUTO ANTÔNIO HOUAISS. Dicionário Houaiss de Sinônimos e Antônimos da Língua Portuguesa. Rio de Janeiro: Objetiva, 2003.

JALILVAND, M. R.; EBRAHIMI, A.; SAMIEI, N. Eletronic word of mouth effects on tourists' attitudes toward islamic destinations and travel intention: an empirical study in Iran. Procedia - Social and Behavioral Sciences, v. 81, p. 484-489, 2013.

JALILVAND, M. R. et al. Examining the structural relationships of eletronic word of mout, destination image, tourist attitude toward destination and travel intention: an integrated approach. Journal of Destination Marketing \& Management, v. 1, p. 134-143, 2012.

KIM, D.-Y.; CHEN, Z. Are people aware of their attitudes toward destination? Understanding the implicit association test in tourism research. Tourism Analysis, v. 15, n. 3, p. 299-313, 2010.

KIM, D.-Y.; CHEN, Z.; HWANG, Y.-H. Are we really measuring what we think we're measuring? assessing attitudes towards destinations with the implicit association test. International Journal of Tourism Research, v. 13, n. 5, p. 468-481, 2011. 
KIM, H.; STEPCHENKOVA, S. Effect of tourist photographs on attitudes towards destination: manifest and latent content. Tourism Management, v. 49, p. 29-41, 2015.

KROSNICK, J. A.; JUDD, C. M.; WITTENBRINK, B. The measurement of attitudes. In:ALBARRACÍN, D.; JOHNSON, B. T.; ZANNA, M. P. (Org.). The handbook of attitudes. Mahwah (New Jersey, EUA): Lawrence Erlbaum Associates, 2005. p. 21-76.

LEE, T. H. A structural model to examine how destination image, attitude, and motivation affect the future behavior of tourists. Leisure Sciences, v. 31, n. 3, p. 215-236, 2009.

LISSITZ, R. W.; GREEN, S. B. Effect of the number of scale points on reliability: A Monte Carlo approach. Journal of Applied Psychology, v. 60, n. 1, p. 10-13, 1975.

MOHSIN, A. Tourist attitudes and destination marketing - the case of Australia's Northern Territory and Malaysia. Tourism Management, v. 26, n. 5, p. 723-732, 2005.

MORAKABATI, Y.; FLETCHER, J.; PRIDEAUX, B. Tourism development in a difficult environment: a study of consumer attitudes, travel risk perceptions and the termination of demand. Tourism Economics, v. 18, n. 5, p. 953-969, 2012.

PHILLIPS, W.; JANG, S. Destination image and tourist attitude. Tourism Analysis, v. 13, p. 401-411, 2008.

RAGHEB, M. G.; TATE, R. L. A behavior model of leisure participation, based on leisure attitude, motivation and satisfaction. Leisure Studies, v. 12, p. 61-67, 1993.

RUNGTUSANATHAM, M.; ANDERSON, J. C.; DOOLEY, K. J. Towards measuring the "SPC implementation/practice" construct: Some evidence of measurement quality. International Journal of Quality \& Reliability Management, v. 16, n. 4, p. 301-329, 1999.

SPARKS, B.; PAN, G. W. Chinese outbound tourists: Understanding their attitudes, constraints and use of information sources. Tourism Management, v. 30, n. 4, p. 483-494, 2009.

UM, S.; CROMPTON, J. L. Attitude determinants in tourism destination choice. Annals of Tourism Research, v. 17, n. 3, p. 432-448, 1990. 